\title{
EXTREME POSITIVE OPERATORS AND HOMOMORPHISMS
}

\author{
BY \\ R. R. PHELPS(1)
}

Consider the following theorem, first proved (in the real case) by Arens and Kelley [1]: Let $X$ be a compact Hausdorff space and $C(X)$ the space of all real (or complex) valued continuous functions on $X$, with the supremum norm. Let $K$ be the convex set of linear functionals $L$ on $C(X)$ such that $L(1)=1=\|L\|$. The set of extreme points of $K$ coincides with the set of all nontrivial multiplicative linear functionals on $C(X)$. Their proof of this result depends on the representation of linear functionals by means of measures (which makes it possible to identify a multiplicative functional with evaluation at a point of $X$ ). Using methods of a more algebraic nature, Tate [8] has proved the above equivalence for certain partially ordered real commutative algebras (which include the algebra $C(X)$ ). The fact that such methods are at all feasible arises, essentially, from the fact that the convex set $K$ admits a second description, namely, it is those linear functionals $L$ on $C(X)$ such that $L \geqq 0$ and $L(1)=1$.

Suppose, now, that we represent the algebra $C(X)$ by $A$ and the algebra of scalars by $B$. The above result then asserts that the set of nontrivial homomorphisms of a certain algebra $A$ into $B$ coincides with the set of extreme points of a certain convex set of linear transformations from $A$ into $B$. The purpose of the present paper is to try to determine the extent to which this type of result remains valid if the algebra of scalars $B$ is replaced by a more general algebra (over the same field as $A$ ). To the best of our knowledge, the first proof of a theorem of this type was given by A. Ionescu-Tulcea and C. Ionescu-Tulcea [5] (announced in [6, footnote 3]), where $A$ and $B$ were taken to be (real) $C(X)$ and $C(Y)$, respectively. Their method is an extension of the method of Arens and Kelley. Working more in the spirit of Tate's paper, and with sets of transformations analogous to the second description of $K$ (above) we will give an extremely simple proof of a similar result (Theorem 1.1) for certain algebras of functions which include algebras of the form $C(X)\left({ }^{2}\right)$. It would be possible to work with

Received by the editors July 25, 1962.

(1) Supported in part by the National Science Foundation. The author wishes to express his gratitude to Professor J. Feldman for numerous conversations on subjects related to the contents of this paper.

(2) We are indebted to the Ionescu-Tulceas for having been permitted to read their manuscript [5]. Although we had previously considered the problem, it was their work which led us to formulate and prove Theorem 1.1. 
certain commutative, partially ordered algebras with unit, but the appropriate hypotheses would invariably bring us back to algebras of functions, so we will restrict our attention to such algebras from the start. More precisely, let $A$ and $B$ be algebras of complex valued functions defined on the sets $X$ and $Y$ respectively, and assume that both $A$ and $B$ contain the constant functions. Let $\mathscr{L}(A, B)$ be the set of all linear transformations from $A$ into $B$, and let $K_{0}(A, B)=\{T: T \in \mathscr{L}(A, B), T \geqq 0$ and $T 1 \leqq 1\}$ (where $T \geqq 0$ means $T f \geqq 0$ whenever $f \in A$ and $f \geqq 0$, and 1 represents the function which equals 1 at each point). Let $K_{1}(A, B)=\left\{T: T \in K_{0}(A, B)\right.$ and $\left.T 1=1\right\}$.

If the functions in $A$ and $B$ are bounded, and these algebras have the supremum norm, let $\mathscr{B} \mathscr{L}(A, B)$ be those $T$ in $\mathscr{L}(A, B)$ such that

$$
\|T\|=\sup \{\|T f\|:\|f\| \leqq 11, f \in A\}<\infty,
$$

and let $K_{2}(A, B)=\{T: T \in \mathscr{B L}(A, B),\|T\|=1$ and $T 1=1\}$. (Whenever this set is introduced, the fact that the functions in $A$ and $B$ are bounded is assumed implicitly.)

We say that $A$ is self-adjoint if $\bar{f} \in A$ whenever $f \in A$ (where $\bar{f}(x)=\overline{f(x)}$ for $x$ in $X)$. Denoting by $A_{R}$ the set of real valued functions in $A$, we see that $A$ is self-adjoint if and only if $A=A_{R}+i A_{R}$.

It is easily seen that each of the above sets $K$ is convex (i.e., $\lambda T_{1}+(1-\lambda) T_{2} \in K$ whenever $T_{1}, T_{2} \in K$ and $\left.0 \leqq \lambda \leqq 1\right)$. An element $T$ of $K$ is an extreme point of $K$ provided $T=\frac{1}{2}\left(T_{1}+T_{2}\right)$ and $T_{1}, T_{2} \in K$ imply $T=T_{1}=T_{2}$. Equivalently, $T$ is an extreme point of $K$ if and only if $U$ in $\mathscr{L}(A, B)$ and $T \pm U \in K$ imply $U=0$. An element $T$ of $\mathscr{L}(A, B)$ is multiplicative, or a homomorphism, if $T(f g)=T f T g$ whenever $f, g \in A$.

Our central result is Theorem 1.1 in which we show that, with an additional assumption on $A$, the extreme points of $K_{0}(A, B)$ are multiplicative. We consider the converse and related questions, and (in \$2) we apply these results to obtain, among other things, the Ionescu-Tulcea theorem. Many of the results concerning the above sets $K$ and their extreme points turn out to depend upon the algebra $A$ being self-adjoint (this being, for instance, precisely the condition under which $\left.K_{2}(A, B)=K_{1}(A, B)\right)$. The following interesting question remains open: Must the extreme points of $K_{2}(A, B)$ be multiplicative? (It is well known that the answer is affirmative if $A$ is a subalgebra of $C(X)$ and $B$ is the algebra of scalars.) An obvious example of a non-self-adjoint algebra on which to test this question is the algebra $\mathscr{A}$ of all functions which are continuous in the closed unit disc in the complex plane and analytic in its interior, with supremum norm. We do not know the answer (with $A=B=\mathscr{A}$ ) even for this special case, although we do have an interesting characterization in $\S 3$ of those homomorphisms of $\mathscr{A}$ into itself which are extreme points of $K_{2}(\mathscr{A}, \mathscr{A})$.

Although all our results are stated and proved for algebras of complex valued 
functions, the analogous results for real algebras of real valued functions are valid, with somewhat simpler proofs.

\section{Basic results.}

THEOREM 1.1. Let $i$ denote 0 or 1 , and suppose that each real valued function in $A$ is bounded. If $T$ is an extreme point of $K_{i}(A, B)$, then $T$ is multiplicative.

Proof. It is easy to verify that any extreme point of $K_{1}(A, B)$ is an extreme point of $K_{0}(A, B)$, so we can restrict our attention to the latter set. We will make repeated use of the fact that if $U \in \mathscr{L}(A, B)$ and $T \pm U \in K_{0}$, then $U=0$. We show first that $T f=T 1 T f$ for all $f$ in $A$. Indeed, let $U f=T f-T 1 T f$. Since $T 1 \leqq 1$, we have $U \geqq 0$, so $T+U \geqq 0$, while $(T-U) f=T 1 T f$ shows that $T-U \geqq 0$. Furthermore, $(T+U) 1=T 1+T 1(1-T 1) \leqq T 1+(1-T 1)=1$ and $(T-U) 1=(T 1)^{2} \leqq 1$. Thus $T \pm U \in K_{0}$, so $U=0$. We show next that $T f g=T f T g$ whenever $g$ is a real valued function in $A$. It suffices to prove this for those $g$ such that $0 \leqq g \leqq 1$, since if $g$ is real valued, it is bounded and there exist positive constants $a$ and $b$ such that $0 \leqq a g+b 1 \leqq 1$. If $T[f(a g+b 1)]=T f T(a g+b 1)$, then $a T f g+b T f=a T f T g+b T f T 1$ and therefore $T f g=T f T g$. Assuming, then, that $0 \leqq g \leqq 1$, let $U f=T f g-T f T g$; we have $U 1=0$ so $(T \pm U) 1=T 1 \leqq 1$. If $f \geqq 0$, then $(T+U) f=T f+T f g-T f T g=T f(1-T g)+T f g_{i} \geqq 0$ since $g \leqq 1$ implies $T g \leqq T 1 \leqq 1$, while $(T-U) f=T f-T f g+T f T g=T[f(1-g)]$ $+T f T g \geqq 0$ since $f(1-g) \geqq 0$. Thus, $U=0$. Finally, to see that $T f g=T f T g$ for any $f, g$ in $A$, choose $f$ and define $U g=T f g-T f T g$. If $g \geqq 0$ (and hence is real valued) the previous result shows that $U g=0$; it follows trivially that $T \pm U \in K_{0}$ and that $T$ is multiplicative.

The hypothesis in the above theorem that every real valued function in $A$ be bounded is (especially in the light of the next result) somewhat strong. We have no example which shows that it is needed; on the other hand, we see no way of proving the theorem without it $\left({ }^{3}\right)$. Although we did not assume that $A$ is self-adjoint, the next result shows that the existence of an extreme point in $K_{0}(A, B)$ implies this fact (and hence our theorem is actually about an algebra of bounded functions). The letter $C$ denotes the set of complex numbers.

Proposition 1.2. If $K_{0}(A, B)$ (or $\left.K_{1}(A, B)\right)$ contains an extreme point, then the algebra $A$ is self-adjoint.

Proof. If there exists $g$ in $A, g \notin A_{R}+i A_{R}$, choose a linear subspace $N$ of $A$

(3) By means of elementary arguments, together with the proof of Theorem 1.1, it is possible to reduce this question to the following: If $A$ is a real algebra of real valued functions on $X$ which contain an unbounded function, must every extreme point of $K_{0}(A, B)$ be multiplicative? A good example of such an algebra (for which we do not know the answer) is the algebra of all real polynomials, considered as functions on the line. It follows from Theorems 1.4 and 1.5 that the multiplicative elements of $K_{0}(A, B)$ are extreme, and it is easily shown that every multiplicative element of $\mathscr{L}(A, A)$ is of the form analogous to that in Theorem 3.4. 
such that $A_{R}+i A_{R} \subset N, N \cap C g=\{0\}$ and $A=N+C g$, where $C g$ denotes the set of all complex multiples of $g$. Define $U$ in $\mathscr{L}(A, B)$ by $U=0$ on $N$ and $U(c g)=c 1$ for each $c$ in $C$. Then if $T \in K_{0}$ we have $T \pm U \in K_{0}$, so that $T$ is not extreme.

As we will see below, the condition that $A$ be self-adjoint turns out to be characteristics of those algebras $A$ for which $K_{1}(A, B) \subset K_{2}(A, B)$.

If $B$ is the algebra of scalars, then $K_{i}(A, B)$ becomes a set of linear functionals, which we abbreviate by $K_{i}(A)$. The analogous set of functionals on $B$ is denoted by $K_{i}(B)$. For each $x$ in $X$ we denote by $L_{x}$ the evaluation functional at $x: L_{x} f=f(x)$ for each $f$ in $A$.

Theorem 1.3. Always, $K_{2}(A, B) \subset K_{1}(A, B)$. These sets are equal if and only if $A$ is self-adjoint.

Proof. To prove the first assertion, it suffices to prove the special case $K_{2}(A) \subset K_{1}(A)$, since, if the latter holds, then for any $T$ in $K_{2}(A, B)$ and $y$ in $Y$ we have $L_{y} \circ T \in K_{2}(A) \subset K_{1}(A)$, so that $(T f)(y)=\left(L_{y} \circ T\right)(f) \geqq 0$ if $f \geqq 0$. Since this is true for each $y$ in $Y$, we have $T \geqq 0$ and therefore $T \in K_{1}(A, B)$. Suppose, then, that $L \in K_{2}(A)$, so that $|L f| \leqq\|f\|$ for all $f$ in $A$. We want to show that $L f \geqq 0$ if $f \geqq 0$. Suppose first, that $L f=a+i b$, with $b \neq 0$. If $\delta$ is real, with $\delta b>0$, then $|L(f+i \delta \cdot 1)|^{2}=a^{2}+(b+\delta)^{2}$, while $|f+i \delta \cdot 1|^{2} \leqq\left(\|f\|^{2}+\delta^{2}\right) \cdot 1$. For sufficiently large $|\delta|$, then, we have $|L(f+i \delta \cdot 1)|^{2}=a^{2}+b^{2}+2 \delta b+\delta^{2}>\|f\|^{2}+\delta^{2}$ $\geqq\|f+i \delta \cdot 1\|^{2}$, a contradiction. Thus, $L f$ must be real. If it were negative, note that since $0 \leqq f \leqq\|f\| \cdot 1$, we have $-\|f\| \cdot 1 \leqq f-\|f\| \cdot 1 \leqq 0$, so that $\|(f-\|f\| \cdot 1)\| \leqq\|f\|$, while $|L(f-\|f\| \cdot 1)|=|L f-\|f\||=\|f\|-L f>\|f\|$, a contradiction which completes the first part of the proof.

Suppose, now, that $A=A_{R}+i A_{R}$. To prove that $K_{1}(A, B) \subset K_{2}(A, B)$, it suffices again to prove the special case $K_{1}(A) \subset K_{2}(A)$. Indeed, suppose that the latter inclusion holds, that $T \in K_{1}(A, B)$ and that $y \in Y$. Then $L_{y} \circ T \in K_{1}(A) \subset K_{2}(A)$, so that for each $f$ in $A,|(T f)(y)|=\left|\left(L_{y} \circ T\right)(f)\right| \leqq\|f\|$; since this is true for each $y$ in $Y$, we conclude that $T \in K_{2}(A, B)$. Suppose, then, that $L \in K_{1}(A)$. If $f \in A_{R}$, then $\|f\| \cdot 1-f \geqq 0$, so $\|f\|-L f=L(\|f\| \cdot 1-f) \geqq 0$, which shows that $L f$ is real. By Tate's argument [8] (he considers the discriminant of the quadratic $0 \leqq L\left[(\lambda f+1)^{2}\right]=\lambda^{2} L\left(f^{2}\right)+2 \lambda L f+1$ in $\left.\lambda\right), L \geqq 0$ implies that $(L f)^{2} \leqq L\left(f^{2}\right)$ for each $f$ in $A_{R}$. Now, for arbitrary $f$ in $A$, we can choose $f_{1}, f_{2}$ in $A_{R}$ such that $f=f_{1}+i f_{2}$, and hence $\|f\|^{2} \cdot 1 \geqq|f|^{2}=f_{1}^{2}+f_{2}^{2}$. It follows that $\|f\|^{2}=L\left(\|f\|^{2} \cdot 1\right) \geqq L\left(f_{1}^{2}\right)+L\left(f_{2}^{2}\right) \geqq\left(L f_{1}\right)^{2}+\left(L f_{2}\right)^{2}=\left|L f_{1}+i L f_{2}\right|^{2}=|L f|^{2}$, so that $\|L\| \leqq 1$.

Finally, we show that if there exists $g$ in $A \sim\left(A_{R}+i A_{R}\right)$, then there exists $T$ in $K_{1}(A, B) \sim K_{2}(A, B)$. We simply choose a subspace $N$ of $A$ such that $A_{R}+i A_{R} \subset N, N \cap C g=\{0\}$, and $A=N+C g$. Choose $x$ in $X$ and $\delta>\|g\|$ and define $T: A \rightarrow B$ by $T(f+c g)=[f(x)+c \delta] \cdot 1$ for $f$ in $N$ and complex $c$. Then $T \in K_{1}(A, B)$, but $\|T g\|=\delta>\|g\|$, so $T \notin K_{2}(A, B)$. 
The nontrivial part of the next theorem is a slight variation of Tate's result [8] for the real case; the proof is also his.

THEOREM 1.4. Let $i$ denote 0 or 1 . Every multiplicative element of $K_{i}(A)$ is an extreme point of $K_{i}(A)$ if (and only if) $A$ is self-adjoint.

Proof. The "only if" part is a consequence of Proposition 1.2 and the fact that $L_{x}$ is a multiplicative element of $K_{i}(A)$, for each $x$ in $X$. Suppose that $L$ is a multiplicative element of $K_{i}(A)$ and that $L=\frac{1}{2}\left(L_{1}+L_{2}\right), L_{1}, L_{2}$ in $K_{i}(A)$. It suffices to show that $L=L_{1}=L_{2}$ on $A_{R}$. Since each $L_{k} \geqq 0$, it follows (as in the proof of Theorem 1.3) that $\left(L_{k} f\right)^{2} \leqq L_{k}\left(f^{2}\right)$ for each $f$ in $A_{R}$; hence, for such $f$, we have $\frac{1}{4}\left(L_{1} f\right)^{2}+\frac{1}{2} L_{1} f L_{2} f+\frac{1}{4}\left(L_{2} f\right)^{2}=(L f)^{2}=L\left(f^{2}\right)=\frac{1}{2} L_{1}\left(f^{2}\right)+\frac{1}{2} L_{2}\left(f^{2}\right)$ $\geqq \frac{1}{2}\left(L_{1} f\right)^{2}+\frac{1}{2}\left(L_{2} f\right)^{2}$, so that $\left(L_{1} f-L_{2} f\right)^{2} \leqq 0$. This shows that $L_{1} f=L_{2} f$ and completes the proof.

The next theorem shows that the converse to Theorem 1.1 for arbitrary $B$ depends on the validity of the converse when $B$ is taken to be the complex numbers.

THEOREM 1.5. Let $i$ denote 0,1 or 2. Every multiplicative element of the set $K_{i}(A, B)$ is an extreme point of this set if and only if the same is true for the set $K_{i}(A)$.

Proof. Suppose that $T$ is a multiplicative element of $K_{i}(A, B)$ and that there exists $U$ in $\mathscr{L}(A, B)$ such that $T \pm U \in K_{i}(A, B)$. If $y \in Y$, then the functional $L_{y} \circ T$ is a multiplicative element of $K_{i}(A)$; by hypothesis it is extreme. Since $L_{y} \circ{ }^{\circ} \pm L_{y} \circ U \in K_{i}(A)$, we see that $L_{y} \circ U=0$. Since this is true for each $y$ in $Y$, we must have $U=0$ and hence $T$ is extreme. To prove the converse, suppose that $L$ is a multiplicative element of $K_{i}(A)$. We define $T$ in $K_{i}(A, B)$ by $T f=L f \cdot 1$ for each $f$ in $A$ (i.e., $T f$ is the constant function $L f$ ). Then $T$ is multiplicative, so (by hypothesis) it is extreme, and one concludes easily that this implies that $L$ is extreme in $K_{i}(A)$.

THEOREM 1.6. Let $i$ denote either 0,1 or 2 , and suppose that $A$ is a selfadjoint algebra of bounded functions. Then an element $T$ of $K_{i}(A, B)$ is multiplicative if and only if it is extreme.

For $i=0$ or 1 , the proof is immediate from Theorems 1.1, 1.5 and 1.4. For $i=2$, we use Theorem 1.3 to reduce the theorem to the case $i=1$.

2. Applications. If $E$ is a subset of $X$, the characteristic function $\chi_{E}$ of $E$ is that function which equals 1 on $E, 0$ on $X \sim E$. A topological space $X$ is said to be totally disconnected if the collection of open and closed subsets of $X$ forms a basis for its topology. The equivalence between (i), (ii) and (iii) in the next theorem was first proved (in the real case, with $B=C(Y)$ ) by $\mathrm{A}$. IonescuTulcea and C. Ionescu-Tulcea [5]. A special case of their theorem has been proved independently by S. Lloyd [7]. 
THEOREM 2.1. Let $X$ and $Y$ be compact Hausdorff spaces, let $A=C(X)$ and let $B$ a subalgebra (containing the constants) of $C(Y)$. Then $K_{1}(A, B)=K_{2}(A, B)$, and the following assertions about an element $T$ of $\mathscr{L}(A, B)$ are equivalent:

(i) $T$ is an extreme point of $K_{1}(A, B)$.

(ii) $T$ is multiplicative and is in $K_{1}(A, B)$.

(iii) There exists a continuous function $\phi: Y \rightarrow X$ such that $T f=f \circ \phi$ for all $f$ in $A$. If $X$ is totally disconnected, the next assertion is also equivalent to the above:

(iv) $T$ carries characteristic functions into characteristic functions and is in $K_{1}(A, B)$.

Proof. The fact that $K_{1}(A, B)=K_{2}(A, B)$ comes from Theorem 1.3 and the fact that $C(X)$ is self-adjoint, while Theorem 1.6 shows that (i) is equivalent to (ii). It is obvious that (iii) implies (ii); to see the converse, suppose that $T$ is multiplicative, that $y \in Y$, and consider $L_{y} \circ T$. This is multiplicative and is in $K_{1}(A)=K_{2}(A)$, so by Theorem 1.4 it is an extreme point of $K_{2}(A)$. By the ArensKelley theorem [1] (see [3, p. 278] for the complex case), there exists a unique point in $X$, which we denote by $\phi(y)$, such that $L_{y} \circ T=L_{\phi(y)}$. This defines $\phi$ for each $y$ in $Y$; to see that $\phi$ is continuous, one uses the fact that the topology of $X$ is the same as the "weak" topology induced on it by $C(X)$, and the fact that $T f$ is continuous on $Y$ for each $f$ in $A$. To see that (ii) implies (iv), suppose that $\chi_{E} \in C(X)$ and that $T$ is a multiplicative element of $K_{i}(A, B)$. Then $T \chi_{E}=T\left(\chi_{E}^{2}\right)=\left(T \chi_{E}\right)^{2}$, so $T \chi_{E}$ must be a characteristic function. Conversely, if $X$ is totally disconnected and $T$ carries characteristic functions into characteristic functions, we will show that $T$ is an extreme point of $K_{1}(A, B)$. Suppose there exists $U$ in $L(A, B)$ such that $T \pm U \in K_{1}(A, B)$. Then for any $\chi_{E}$ in $A$, we have $0 \leqq \chi_{E} \leqq 1$, so that $0 \leqq T \chi_{E} \pm U \chi_{E} \leqq 1$. Since $T \chi_{E}$ only takes on the values 0 and 1 , we conclude that $U \chi_{E}=0$. Thus, $U=0$ on the subspace of $A$ spanned by the characteristic functions. Now, this subspace is in fact a subalgebra, and since $X$ is a totally disconnected Hausdorff space, the characteristic functions separate points of $X$. It follows from the Stone-Weierstrass theorem [3] that this subspace is dense in $A$. Furthermore, $T \in K_{2}(A, B)$ and $T+U \in K_{2}(A, B)$ show that $U$ is continuous, so $U=0$ on $A$ and the proof is complete.

Parts (i), (ii) and (iv) of the above theorem are valid if we replace $K_{1}(A, B)$ by $K_{0}(A, B)$; the appropriate formulation of (iii) becomes a little more complicated, since the map $\phi$ is only defined on those $y$ in $Y$ for which $(T 1) y=1$. By using the method of proof of Theorem 1.3, one sees that $K_{0}(A, B)$ $=\{T: T \in \mathscr{L}(A, B),\|T\| \leqq 1$ and $0 \leqq T 1 \leqq 1\}$.

Note that the hypotheses on $A$ in Theorem 1.6 imply that if $A$ is given the supremum norm, that it is isometric and isomorphic with a dense subalgebra of $C(Z)$ for some compact Hausdorff space $Z$ [3]. Thus, it is possible to deduce Theorem 1.6 by means of the above results.

In proving the equivalence of (i) and (iv) in Theorem 2.1, the hypothesis that 
$X$ be totally disconnected was only used in showing that (iv) implies (i). This implication is not valid otherwise $\left({ }^{4}\right)$; indeed, suppose $X$ were not totally disconnected. Then an elementary argument shows that there would exist a connected component $E$ of $X$ containing at least two distinct points $x_{1}$ and $x_{2}$. Define $T f=\frac{1}{2}\left[f\left(x_{1}\right)+f\left(x_{2}\right)\right] \cdot 1$, for each $f$ in $C(X)$. If $f=\chi_{F}$ for some subset $F$ of $X$, then, since $E$ is connected, either $f=0$ or $f=1$ on $E$, so that $T f=0$ or 1 and hence is certainly a characteristic function. Clearly, $T \in K_{1}(A, B)$, although it is not extreme in this set, since $T=\frac{1}{2}\left(T_{1}+T_{2}\right)$, where $T_{i}$ is the element of $K_{1}(A, B)$ defined by $T_{i} f=f\left(x_{i}\right) \cdot 1, i=1,2$.

We denote by $L^{\infty}(S, \Sigma, \mu)$ the space of all essentially bounded measurable functions on the positive measure space $(S, \Sigma, \mu)$, with essential supremum norm.

THEOREM 2.2. Let $A=L^{\infty}\left(S_{1}, \Sigma_{1}, \mu_{1}\right)$ and suppose that $B$ is a subalgebra (containing the constants) of $L^{\infty}\left(S_{2}, \Sigma_{2}, \mu_{2}\right)$. Then $K_{0}(A, B)=\{T: T \in \mathscr{L}(A, B)$, $\|T\| \leqq 1$ and $0 \leqq T 1 \leqq 1\}$ and the following assertions about an element $T$ of $K_{0}(A, B)$ are equivalent:

(i) $T$ is an extreme point of $K_{0}(A, B)$.

(ii) $T$ is multiplicative.

(iii) $T$ carries characteristic functions into characteristic functions.

The proof follows from the proof of Theorem 2.1 (and the subsequent remarks), together with the well-known fact that there exist extremely disconnected (hence totally disconnected) compact Hausdorff spaces $X_{i}$ such that $L^{\infty}\left(S_{i}, \Sigma_{i}, \mu_{i}\right)$ is isometrically isomorphic with $C\left(X_{i}\right), i=1,2[3, \mathrm{p} .445]$.

3. An example. The first three results of this section deal with an algebra $A$ of bounded complex valued functions on a set $X$, with supremum norm. After that we consider the algebra $\mathscr{A}$ of all complex valued functions which are defined and continuous in $|z| \leqq 1$ and analytic in $|z|<1$, with supremum norm. By the unit ball of one of these spaces, we mean the set of all $f$ of norm at most 1 .

Lemma 3.1. Suppose $f \in A,\|f\| \leqq 1$. Then $f$ is not an extreme point of the unit ball of $A$ if and only if there exists $g$ in $A, g \neq 0$, such that $|f|+|g| \leqq 1$.

$\operatorname{Proof}\left({ }^{5}\right)$. If such a $g$ exists, then $|f \pm g| \leqq|f|+|g| \leqq 1$, so $\|f \pm g\| \leqq 1$ and $f$ is not extreme. Conversely, if $\|f \pm h\| \leqq 1, h \neq 0$, then for each $x$ in $X$ we have

$$
1 \geqq|f(x) \pm h(x)|^{2}=|f(x)|^{2} \pm 2 \mathscr{R} f(x) \overline{h(x)}+|h(x)|^{2}
$$

and therefore $|f|^{2}+|h|^{2} \leqq 1$. Thus, $|h|^{2} \leqq 1-|f|^{2}=(1-|f|)(1+|f|) \leqq 2(1-|f|)$, so $|f|+\frac{1}{2}|h|^{2} \leqq 1$ and we can let $g=\frac{1}{2} h^{2}$.

Corollary 3.2. Suppose $f \in A,\|f\| \leqq 1$. Then $f$ is an extreme point of the unit ball if and only if $f^{n}$ is an extreme point, $n=1,2,3, \cdots$.

(4) This fact was proved in a conversation with W. G. Bade.

(5) This proof differs slightly from one originally suggested to us by Eva Kallin. 
Proof. If $f^{n}$ is not extreme, there exists $g \neq 0$ such that $|f|^{n}+|g| \leqq 1$. Then

$$
|g| \leqq 1-|f|^{n}=(1-|f|)\left(1+|f|+|f|^{2}+\cdots+|f|^{n-1}\right) \leqq n(1-|f|),
$$

so $|f|+(1 / n)|g| \leqq 1$ and hence $|f|$ is not extreme. Conversely, if there exists $g \neq 0$ such that $|f|+|g| \leqq 1$ then $|f|^{n} \leqq|f|$ shows that $|f|^{n}+|g| \leqq 1$, so that $f^{n}$ is not extreme.

Corollary 3.3. Suppose $|f| \in A$ whenever $f \in A$. Then $f(\|f\| \leqq 1)$ is an extreme point of the unit ball of $A$ if and only if $|f|=1$.

Proof. If $|f(x)|<1$ for some $x$ in $X$, then $g=1-|f| \neq 0$ and $|f|+|g| \leqq 1$. Conversely, if there exists $g \neq 0,|f|+|g| \leqq 1$, then $|f(x)|<1$ for some $x$.

The remainder of this section is devoted to the algebra $\mathscr{A}$ defined above. Since the only real valued functions in $\mathscr{A}$ are real constants, it follows from Proposition 1.2 that the sets $K_{0}(\mathscr{A}, \mathscr{A})$ and $K_{1}(\mathscr{A}, \mathscr{A})$ have no extreme points. Whether the extreme points of $K_{2}(\mathscr{A}, \mathscr{A})$ are multiplicative remains an open question; we will describe the homomorphisms of $\mathscr{A}$ into itself, and determine which of these are extreme in $K_{2}(\mathscr{A}, \mathscr{A})$. The following result uses no new methods, but we know of no reference to it in the literature. The function in $\mathscr{A}$ which sends $z$ into $z$ is denoted by $I$.

THEOREM 3.4. Suppose that $g \in \mathscr{A},\|g\| \leqq 1$, and define (for each $f$ in $\mathscr{A}$ ) $T f=f \circ g$. Then $T$ is a multiplicative element of $K_{2}(\mathscr{A}, \mathscr{A})$, with $T I=g$. On the other hand, if $T$ is a nontrivial multiplicative element of $\mathscr{L}(\mathscr{A}, \mathscr{A})$, then $T \in K_{2}(\mathscr{A}, \mathscr{A}),\|T I\| \leqq 1$ and $T f=f \circ T I$ for all $f$ in $\mathscr{A}$.

Proof. If $T f=f \circ g$, the properties stated above are easily verified. Suppose, then, that $T$ is a nontrivial multiplicative transformation. Since $T 1=(T 1)^{2}$, and since $|z| \leqq 1$ is connected, we must have $T 1=0$ or $T 1=1$. The former would imply that $T=0$, so $T 1=1$. If $|z| \leqq 1$, then $L_{z} \circ T$ is a homomorphism of $\mathscr{A}$ onto the complex numbers and hence is continuous, with norm 1 . Thus, for each $f$ in $\mathscr{A}$ and $|z| \leqq 1$ we have $|(T f)(z)|=\left|\left(L_{z} \circ T\right) f\right| \leqq\|f\|$, so that $\|T f\| \leqq\|f\|$ and hence $T \in K_{2}(\mathscr{A}, \mathscr{A})$. Since $\|I\|=1$, we have $\|T I\| \leqq 1$. Finally, suppose that $f=\sum_{k=0}^{n} a_{k} I^{k}$ is a polynomial in $\mathscr{A}$; then $T f=\sum a_{k}(T I)^{k}$ $=f \circ T I$. By continuity of $T$ and density of the polynomials in $\mathscr{A}$, we get the desired result.

THEOREM 3.5. Suppose $T$ is a homomorphism from $\mathscr{A}$ into $\mathscr{A}$ (so that $T f=f \circ T I$ for each $f$ in $\mathscr{A})$. Then $T$ is an extreme point of $K_{2}(\mathscr{A}, \mathscr{A})$ if and only if $T I$ is an extreme point of the unit ball of $\mathscr{A}$.

Proof. Suppose $h=T I$ is extreme and suppose there exists $U$ in $\mathscr{L}(\mathscr{A}, \mathscr{A})$ such that $T \pm U \in K_{2}(\mathscr{A}, \mathscr{A})$. If $f \in \mathscr{A},\|f\| \leqq 1$, we have $\|T f \pm U f\|=\|f \circ h \pm U f\|$ 
$\leqq 1$; in particular, taking $f=I^{n}, n=0,1,2, \cdots$, we have $\left\|h^{n} \pm U I^{n}\right\| \leqq 1$. By Corollary 3.2, $h^{n}$ is an extreme point of the unit ball for $n=1,2,3, \cdots$, while $1=h^{0}$ is extreme by Lemma 3.1. It follows from Lemma 3.1 that $U I^{n}=0$ for each $n$, and hence $U=0$ on all polynomials. Since $U$ is continuous and the polynomials are dense in $\mathscr{A}, U=0$ on $\mathscr{A}$ and $T$ is extreme.

To prove the converse, suppose $h$ is not an extreme point of the unit ball of $\mathscr{A}$. By Lemma 3.1, there exists $g \neq 0$ in $\mathscr{A}$ such that $|h|+|g| \leqq 1$. Define, for $f$ in $\mathscr{A}, U f=\frac{1}{4} f^{\prime}(0) g$; clearly $U$ is a nontrivial element of $\mathscr{L}(\mathscr{A}, \mathscr{A})$ and $U 1=0$. It remains to show that $\|T \pm U\| \leqq 1$, i.e., if $\|f\| \leqq 1$ and $|z|<1$, then $\left|f(h(z)) \pm \frac{1}{4} f^{\prime}(0) g(z)\right| \leqq 1$. We will use the following corollaries to Schwartz's lemma (see, e.g., [2, p. 18]): If $f \in \mathscr{A},\|f\| \leqq 1$ and $|w|<1$, then $\left|f^{\prime}(0)\right|$ $\leqq 1-|f(0)|^{2}$ and

$$
|f(w)| \leqq[|w|+|f(0)|] \cdot[1+|w||f(0)|]^{-1} .
$$

For our purposes we will take $w=h(z)$, and let $a=|h(z)|, b=|f(0)|$. Thus, using the above two inequalities as well as $|g(z)| \leqq 1-|h(z)|$, we have $\left|f(h(z)) \pm \frac{1}{4} f^{\prime}(0) g(z)\right| \leqq|f(h(z))|+\frac{1}{4}\left|f^{\prime}(0)\right||g(z)| \leqq(a+b)(1+a b)^{-1}+\frac{1}{4}\left(1-b^{2}\right)(1-a)$.

Now, $1+b \leqq 2$, so the quantity on the right is at most

$$
\begin{aligned}
(a+b)(1+a b)^{-1}+\frac{1}{2}(1-b)(1-a) & =\left[(a+b)+(1+a b) \cdot \frac{1}{2}(1-b)(1-a)\right](1+a b)^{-1} \\
& \leqq[(a+b)+(1-b)(1-a)](1+a b)^{-1}=1,
\end{aligned}
$$

since $1+a b \leqq 2$. This completes the proof.

It should be noted that the second half of this proof is valid for the algebra $H^{\infty}$ [4]; we need only assume that $h$ is not a constant of modulus one, so that $f \circ h$ is defined for $f$ in $H^{\infty}$. The proof of the first part (as well as the second half of Theorem 3.4) fails for $H^{\infty}$, however, since the polynomials are not dense in this algebra.

Added in proof. Let $A$ be an algebra of bounded complex-valued functions on a set $X$, and suppose that $A$ is complete in the supremum norm. If $g \in A$, $\|g\| \leqq 1, f \in \mathscr{A}$ and $0<r<1$, then (as is well known) $f_{\circ}(r g) \in A$. It follows from the completeness of $A$ and the uniform continuity of $f$ that $\lim _{r \rightarrow 1} f \cdot(r g)$ exists in $A$ and equals $f \circ g$. This fact, together with minor modifications in the proofs of the last two theorems, proves the following result.

THEOREM 3.6. An element $T$ of $K_{2}(\mathscr{A}, A)$ iis multiplicative if and only if there exists $g$ in $A,\|g\| \leqq 1$, such that $T f=f \circ g$ for each $f$ in $\mathscr{A}$. Such a homomorphism is an extreme point of $K_{2}(\mathscr{A}, A)$ if and only if $g$ is an extreme point of the unit ball of $A$.

Finally, we note that characterizations of the extreme points of the unit balls of $\mathscr{A}$ and of $H^{\infty}$ appear in [4, p. 139]. 


\section{BIBLIOGRAPHY}

1. R. F. Arens and J. L. Kelley, Characterizations of the space of continuous functions over a compact Hausdorff space, Trans. Amer. Math. Soc. 62 (1947), 499-508.

2. C. Carathéodory, Theory of functions, Vol. 2, Chelsea, New York, 1954.

3. N. Dunford and J. T. Schwartz, Linear operators. I, Interscience, New York, 1958.

4. K. Hoffman, Banach spaces of analytic functions, Prentice-Hall, Englewood Cliffs, N. J., 1962.

5. A. Ionescu-Tulcea and $\mathrm{C}$. Ionescu-Tulcea, $A$ note on extreme points (unpublished).

6. - On the lifting property. I, J. Math. Anal. Appl. 3 (1961), 537-546.

7. S. Lloyd, On extreme averaging operators. Proc. Amer. Math. Soc. 14 (1963), 305-310.

8. J. Tate, On the relation between extremal points of convex sets and homomorphisms of algebras, Comm. Pure Appl. Math. 4 (151), 31-32.

\section{UNIVERSITY OF CALIFORNIA, \\ Berkeley, California}

